\title{
«Ich bin 1 Volk ». Chance 2000 : subversion et renaissance de l'espace public chez Christoph Schlingensief
}

"Ich bin 1 Volk“. Chance 2000: Subversion und Neubelebung des öffentlichen Raums bei Christoph Schlingensief

"Ich bin 1 Volk". Chance 2000: Christoph Schlingensief's Subversion and Rebirth of Public Space

\section{Emmanuel Béhague}

\section{OpenEdition}

Journals

Édition électronique

URL : https://journals.openedition.org/ceg/8676

DOI : $10.4000 /$ ceg. 8676

ISSN : 2605-8359

Éditeur

Presses Universitaires de Provence

Édition imprimée

Date de publication : 1 mai 2013

Pagination : 225-241

ISBN : 0751-4239

ISSN : 0751-4239

Référence électronique

Emmanuel Béhague, « «Ich bin 1 Volk». Chance 2000 : subversion et renaissance de l'espace public chez Christoph Schlingensief », Cahiers d'Études Germaniques [En ligne], 64 | 2013, mis en ligne le 02 janvier 2020, consulté le 02 juin 2021. URL : http://journals.openedition.org/ceg/8676 ; DOI : https:// doi.org/10.4000/ceg.8676 


\title{
«Ich bin 1 Volk ». Chance 2000 : subversion et renaissance de l'espace public chez Christoph Schlingensief
}

\author{
Emmanuel BÉHAGUE \\ Université de Strasbourg
}

En mars 1998, dans le contexte de la campagne électorale en vue des élections au Bundestag du 27 septembre, Christoph Schlingensief donne naissance à un projet pour le moins difficile à saisir à partir de l'outillage terminologique usuel dans le domaine du théâtre, étalé dans le temps car composé d'un grand nombre d'interventions publiques jusqu'au soir des résultats, et dans l'espace, dans la mesure où celles-ci se déroulent en divers lieux, théâtraux ou non-théâtraux. Ensemble, elles forment une continuité aussi cohérente qu'hétérogène dont l'amorce est la fondation officielle du parti politique Chance 2000. Avec Chance 2000, l'en-dehors du théâtre devient le terrain d'un jeu en plusieurs parties, aux règles différentes à chaque fois, mais toujours savamment orchestrées par un artiste qui, malgré la dimension aléatoire qui caractérise ses réalisations', demeure l'organisateur minutieux des événements et de leur déroulement. Le premier, intitulé Wahlkampfzirkus, est une série de soirées organisées au Prater avec la troupe du cirque Sperlich, sous un chapiteau, et constituant un mélange hétéroclite de performances, de débats, d'interventions spontanées du public. Hotel Prora. Übernachten bei Chance 2000 an der Volksbühne Berlin, du 15 au 23 mai 1998, pourrait être lu comme une sorte de contre-modèle au Loft de la télé-réalité, un format avec lequel Schlingensief jouera plus tard dans sa célèbre action Bitte liebt Österreich à l'occasion des Wiener Festwochen (2000). Durant l'été, est par ailleurs menée une campagne électorale faite de manifestations organisées à travers l'Allemagne, souvent en lien soit avec un

1 Outre les contributions ćclairantes qu'il rasscmble, préscntées lors d'un symposium organisć à Viennc du 6 au 10 avril 2011, l'ouvrage Der Gesamtkünstler Christoph Schligensief, dirigć par Pia Janke et Teresa Kovacs, proposc un panorama chronologique sommaire mais complet de ces réalisations, ainsi qu'une riche documentation iconographique [Pia JANKE, Teresa KOVACS (dir.), Der Gesamtkïnstler Christoph Schlingensief, Wien, Praesens Verlag, $2011]$. 
théâtre, soit avec une manifestation théâtrale. Un des moments forts en est le bain collectif organisé dans le Wolfgangsee le 2 août 1998 qui fera l'objet plus loin d'une analyse détaillée. À cela s'ajoute une activité permanente à Berlin sous la forme de placardages, de défilés, de rencontres avec le public, avant, au soir des élections, une dernière manifestation intitulée Wahlkampfdebakel' '98 à la Volksbühne. Ces quelques exemples mettent en évidence une volonté de placer ou de replacer le théâtre dans l'espace public, notion ici conçue dans son double sens.

Le premier est celui que lui donne l'urbanisme, espace physique, espace de circulation des hommes et des idées, espace identitaire également. À Berlin, Schlingensief investit quelques uns de ces espaces, savamment choisis. Ainsi, la Kastanienallee, où se trouve le Prater, est-elle le lieu d'une culture alternative avant et après 1989, symbole au milieu des années 1990 d'une forme d'interrègne, moment de suspension entre le passé est-allemand et l'avenir radieux que promet la gentrification progressive de Prenzlauer Berg, biotope de ce que Diedrich Diederichsen appelle « la seule subculture plus ou moins réunifiée d'Allemagne $»^{2}$; Kreuzberg abrite la contestation et la contre-culture ouest allemande des années 1980 ; le Kadewe (Kaufhaus des Westens), enfin, fut la vitrine de la consommation occidentale, à tel point qu'il apparaissait dans les guides touristiques à côté de la Gedächtniskirche ou du Zoologischer Garten. Ces lieux sont donc ceux que parcourent Schlingensief et son équipe : acteurs professionnels, dramaturges, assistants, mais aussi des personnes handicapées qui ont, pour certaines, suivi le metteur en scène tout au long de son parcours, et les laissés-pour-compte de la société allemande venus se joindre au mouvement. L'espace public dont il est question ici est aussi l'espace, non-physique cette fois, de communication entre la sphère privée et l'État, espace dont Jürgen Habermas a analysé la formation ${ }^{3}$. Depuis sa naissance au $\mathrm{XVIII}^{\mathrm{e}}$ siècle, il est pris dans une dynamique constante de transformation dont l'avènement contemporain de la médiatisation constitue une étape.

L'objet de cette contribution sera de montrer de quelle manière Christoph Schlingensief, avec Chance 2000, opère la jonction entre ces deux définitions de l'espace public en le réinvestissant par un mouvement de subversion paradoxal, dans la mesure où il doit contribuer non à sa remise en cause critique mais à sa régénération politique. On s'interrogera donc tout d'abord sur ce qui fait la particularité du geste artistique qu'est Chance 2000, c'est-àdire son caractère de mouvement politique et son autodéfinition comme parti, avant de montrer de quelle manière c'est précisément la nature paradoxale de

2 «Dic cinzige cinigermaßen wiedervereinigte Subkultur Deutschlands ». Diedrich DIEDERICHSEN, « Magic und Massenarbeitslosigkeit: Christoph Schlingensief , Chance 2000' im ,Prater' in Prenzlauer Berg ", in Josef BIERBICHLER, Christoph SCHLINGENSIEF, Harald MARTENSTEIN, Engagement und Skandal. Ein Gespräch, Berlin, Alexander Verlag, 1998 , p. 70 1962.

3 Jürgen HABERMAS, Strukturwandel der Öffentlichkeit, Frankfurt am Main, Suhrkamp, 
l'entreprise - un parti qui n'en est pas un - qui en fait le moteur de la subversion. Quelques actions conçues et réalisées par Schlingensief seront enfin interprétées comme autant de réponses aux mutations contemporaines de l'espace public, dans le sens d'une aspiration à faire renaître celui-ci.

\section{Un parti en creux}

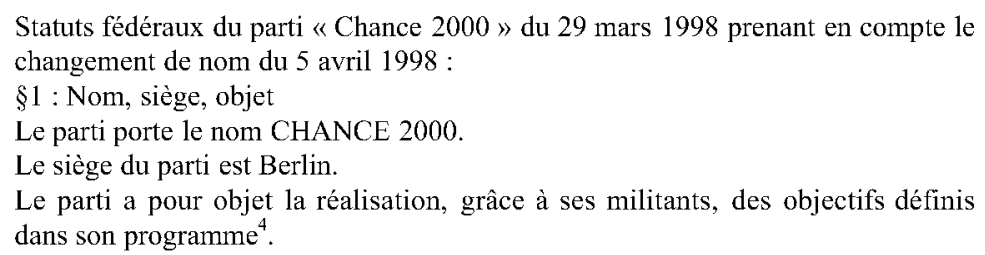

L'affirmation selon laquelle les champs politique et artistique se superposeraient dans l'œuvre de Christoph Schlingensief relève de l'évidence. Ses différents travaux, du fait du contexte de leur émergence et de la possibilité même de les rattacher à tel ou tel genre (film, installation, performance, mises en scène) $)^{5}$ sont néanmoins abordés comme des productions artistiques à dimension politique. Chance 2000, de ce point de vue, articule différemment les deux plans. S'il manie avec brio toutes les formes de l'humour, le metteur en scène ne laisse pas de souligner que tout ceci est sérieux. Il convient dès lors d'appréhender l'objet d'étude en partant de la définition qu'il se donne, à savoir un parti politique. Le Lexique de science politique synthétise de la manière suivante les différentes approches de l'objet : «Les partis politiques sont des entreprises dont l'objectif est la conquête du pouvoir par la mobilisation de soutiens électoraux»". De cette définition, aussi générale qu'elle soit, ressort d'emblée le constat selon lequel Chance 2000 n'est pas un parti politique, dans la mesure où la conquête et l'exercice du pouvoir ne constituent pas ses objectifs. Certes, un calendrier publié dans l'ouvrage «Wähle Dich selbst », édité par le dramaturge Carl Hegemann et Schlingensief lui-même, retrace à rebours les étapes d'une fictive ascension vers la victoire :

4 « Bundessatzung der Partei ,Chance 2000' vom 29.3.1998 i. V. m. Namensänderung v. 5.4. 1998

§1: Name, Sitz und Zweck

Die Partei führt den Namen CHANCE 2000.

Sitz der Partei ist Berlin.

Die Partei hat den Zweck, mit Hilfe der Mitglieder die Ziele des Parteiprogramms zu verwirklichen. 》

Document disponible sur le site du Landesverband Chance 2000 Baden-Württemberg: http://parteien.freepage.de/chance_2000/mort/mort.html (consulté le 30 octobre 2012).

5 Cette affirmation ne revient bien évidemment pas à nier la remise en cause des définitions de telle ou telle forme comme constante esthétique et théorique dans l'œuvre de Schlingensief.

6 Olivier NAY (dir.), Lexique de science politique : vie et institutions politiques, Paris, Dalloz, p. 385. C'est moi qui souligne. 
Treize étapes vers la victoire

Un scénario catastrophe très hypothétique (depuis les élections jusqu'à maintenant. À lire en commençant par la fin !) : 14.27 septembre : aux élections fédérales, Chance 2000 dépasse légèrement la barre des $50 \%$ et entre au Bundestag avec Schlingensief, désigné pour devenir Chancelier (et maintenant ?), chœurs de deuil et chœurs brechtiens sur toutes les chaînes. Pour chaque suffrage, le parti reçoit 1 DM de l'État, soit 30 millions de Mark au total ! (si cependant il n'avait obtenu que $0,5 \%$ de tous les suffrages, il n'aurait rien eu). ${ }^{7}[\ldots]$

Néanmoins, le parti - c'est-à-dire Schlingensief lui-même - délimite de manière restrictive le champ de son action : sa fonction doit demeurer celle de déclencheur, tout au plus de moteur d'un mouvement plus large d'affirmation de soi des exclus (chômeurs, travailleurs précaires, personnes âgées, handicapés...), selon un principe que l'équipe dramaturgique qualifie de rhizome ${ }^{8}$. Les caractéristiques de ce modèle épistémologique transversal correspondent en effet à la dynamique que le parti est censé initier :

Contre les systèmes centrés (même polycentrés), à communication hiérarchique et liaisons préétablies, le rhizome est un système acentré, non hiérarchique et non signifiant, sans Général, sans mémoire organisatrice ou automate central, uniquement défini par une circulation d'états'.

Ainsi défini, le rhizome ne connaît pas de terme, et le pouvoir ne peut donc pas constituer une fin. Sa conquête apparaît bien dans le programme que se donne le parti réuni en congrès le 27 mars 1998, à l'article 3, intitulé Der Wille zur Macht (La Volonté de puissance) en référence à Nietzsche. Mais la notion y prend un sens nouveau :

Chance 2000 aspire au pouvoir, à savoir l'emploi et la mise en pratique politique des potentiels que chacun des utilisateurs libère en faisant usage du réseau interactionnel que procure Chance 2000. Le pouvoir et l'espace opérationnel de Chance 2000 découlent du fait que les membres auxquels il s'adresse des

7 « Dreizehn Schritte zum Sieg

Ein sehr spekulatives Katastrophen-Szenario (von der Wahl bis heute. Von hinten nach vorne lesen!):

14. 27. September: Chance 2000 erreicht bei der Bundestagswahl knapp über $50 \%$ und zieht mit dem designierten Kanzler Schlingensief in den Bundestag ein (was nun?), Trauerchoral und Brechtchoral auf allen Kanälen. Die Partei erhält für jede Stimme $1 \mathrm{DM}=$ ca. 30 Millionen Mark vom Staat! (wenn sie allerdings weniger als 0,5\% aller Stimmen bekommen hätte, hätte sie gar nichts gekriegt). [...]»

Carl HEGEMANN, "Katastrophen-Szenario », in Christoph SCHLINGENSIEF, Carl HEGEMANN (Hg.), Chance 2000. Wähle Dich selbst, Köln, Verlag Kiepenheuer \& Witsch, 1998, p. 44

8 Voir par exemple le texte «Pilzgeflecht (Rhizom) », in ibid., p. 84-86.

9 Gilles DELEUZE, Félix GUATTARI, Rhizome, Paris, Editions de Minuit, 1976, p. 62. 
différentes minorités passent du statut d'objets à celui de sujets de la politique grâce au programme d'humanité défendu par Chance $2000^{10}$.

Chance 2000 conserve donc une catégorie centrale dans la définition du parti politique, mais pour mieux la redéfinir. L'accession au pouvoir signifie l'accession collective au pouvoir d'agir politiquement. Le pouvoir ne se conserve pas, il est au contraire un état dans lequel le parti lui-même perd sa raison d'être.

Second élément mis en évidence dans la définition du parti, la nécessaire présence de "soutiens électoraux » pose également problème. La campagne électorale de Chance 2000, si on accepte le principe de sa réalité en tant que telle, vise en effet à rassembler non pas tant des votants que des candidats. Le mot d'ordre le plus récurrent, Wähle Dich selbst (" Vote pour toi-même »), repris sur de nombreux supports, signifie de facto la suppression de la distinction entre électeurs et candidats. Il ne s'agit pas de recueillir l'adhésion électorale à un projet de société, mais de (re)valoriser l'individu en tant que sujet politique à un degré qui se situe "en deçà » même de son statut d'électeur, c'est-à-dire en tentant de faire renaître chez lui la conscience de son identité sociale.

Cet aspect nous conduit du reste également à contester à Chance 2000 le statut de parti en fonction de la suite de la définition précédemment citée : "Les partis politiques sont des lieux de représentation politique, de production idéologique, de mobilisation des soutiens et de sélection du personnel politique ${ }^{11}$. $\gg$ Dès lors que la raison d'être du parti est d'encourager l'individu à affirmer son existence politique, il ne peut y avoir de sélection du personnel politique, tout comme devient caduque la notion de représentation. Ainsi, le Wahlkampfzirkus inauguré le 13 mars 1998 au Prater, qui est désigné par les organisateurs comme le siège du parti, est-il un lieu ouvert, où chacun est libre d'exposer ses idées, ses propositions. Plus largement, tous sont invités à participer au mouvement par une «action » concrète, quelle qu'elle soit.

Enfin, la référence à Max Weber, qui fait du parti une forme d'« entreprise politique », ne permet pas non plus d'éclairer réellement l'objet considéré :

Nous entendons par entreprise [Betrieb] une action continue en finalité [kontinuierliches Handeln] et par groupement organisé en entreprise

10 «Chance 2000 will die Macht, und zwar die Nutzung und politische Umsetzung der Potentiale, die die einzelnen User durch Gebrauch des von Chance 2000 vermittelten Interaktionsnetzes freisetzen. Die Macht und der politische Operationsraum von Chance 2000 leitet sich daraus her, dass die von ihr anvisierten Menschen der verschiedenen Minderheiten durch das von Chance 2000 propagierte humane Programm von Objekten zu Subjekten der Politik werden.» Site internet du Landesverband Chance 2000 Baden-Württemberg (note 4). Ce même programme apparaît dans l'ouvrage Chance 2000. Wähle Dich selbst (note 7), avec une différence : le parti y est toutefois dénommé Chance 2000. PLC (« Partei der Letzten Chance »), nom qu'il se donne au terme du congrès du 5 avril 1998.

11 Olivier NAY (dir.), Lexique de science politique, p. 385-386. 


\begin{abstract}
[Betriebsverband] unc sociation comportant unc dircction administrative à caractère continu, agissant en finalite ${ }^{12}$.

Nous appclons sociation [Vergesellschaftung] unc rclation socialc lorsquc, ct tant que, la disposition de l'activité sociale se fonde sur un compromis [Ausgleich] d'intérêts motivé rationnellement (cn valeur ou en finalité) ou sur unc coordination [Verbindung] d'intérêts motivéc de la même manic̀rc ${ }^{13}$.
\end{abstract}

Cette notion de «sociation » doit être employée de manière nuancée dans le cas de Chance 2000. Nous avons ici affaire à une relation sociale fondée sur la recherche du débat et en fonctions de valeurs. Néanmoins, le principe d'une « coordination d'intérêts » paraît problématique, dès lors que la valorisation de l'individu et de sa biographie est au centre du programme politique et non pas la recherche d'une fédération d'intérêts qui seraient propres à une communauté, terme employé ici à dessein, dans la mesure où il devra être opposé plus loin à la notion d'espace public.

L'objet Chance 2000 apparaît donc sous un jour éminemment paradoxal. $\mathrm{Du}$ fait de sa visibilité ainsi que de la continuité de son existence, même brève, on ne saurait voir dans le mouvement qu'une simple suite d'actions ou de performances. Cependant, son fonctionnement et sa raison d'être ne satisfont pas, ou de manière partielle et ambiguë seulement, aux critères d'identification comme parti tels que l'aspiration à accéder au pouvoir et à le conserver, et la recherche d'adhésion et de soutiens par les suffrages.

Ce paradoxe est d'autant plus frappant lorsque l'on considère l'ensemble de l'appareil mis en branle pour se donner les formes d'un parti politique. Il faut ainsi relever l'emploi systématique du vocabulaire de la concurrence électorale dans les différents supports assurant la promotion de l'entreprise ; parmi les manifestations qui constituent celle-ci, les performances organisées durant l'été (du $1^{\mathrm{er}}$ juin au 31 juillet, puis du 10 au 25 septembre 1998) dans divers festivals et théâtres en Allemagne et en Autriche sont rassemblées sous le titre Wahlkampftournee; enfin, la terminologie censée décrire le fonctionnement du parti relève strictement du politique : il est question, dans les textes, de Mitglieder (« membres ») de Mitgliederversammlung, (« assemblée des membres ») de Parteizentrale («centrale du parti ») de Landesliste ( liste régionale »)... L'identité politique $\mathrm{du}$ mouvement est donc le produit d'une construction discursive.

Plus frappant encore est le souci de conférer au projet une forme juridique claire et minutieuse. Ainsi, les statuts du parti sont clairement définis dans le document en date du 29 mars 1998 cité plus haut. Un document spécifique (Schiedsordnung), voté en congrès le 14 avril 1998, décrit en 17 articles les motifs possibles de destitution et d'exclusion ainsi que les procédures juridiques à mettre en œuvre ${ }^{14}$. Enfin, l'association Chance 2000, support du

12 Max WEBER, Économie et société /I. Les catégories de la sociologie, Paris, Plon, 1991 (1921), p. 94.

13 Ibid., p. 78

14 Voir note 4. 
parti, dispose elle-même de statuts conformes, rédigés le 16 février 1998 par Carl Hegemann ${ }^{15}$.

Le document-clé, qui vient parfaire l'édifice, est le programme déjà évoqué. Son préambule, curieusement intitulé alteuropäische Präambel (« préambule de la vieille Europe »), met clairement en évidence la notion de dignité humaine, mais également un souci sincère pour l'avenir du pays :

Les fissures dans la structure sociale qui s'approfondissent du fait d'un chômage croissant, ainsi que la perte de dignité humaine accompagnant l'augmentation effrénée des exclusions sociales, exigent des réorientations politiques, sociales, mais aussi dans notre comportement quotidien, que ne peuvent initier euxmêmes les partis qui ont jusqu'à présent pris part à l'élaboration politique de la société. Soucieux d'un avenir socialement et politiquement stable pour notre pays dans le prochain millénaire, Chance 2000 se définit comme un parti offrant une aide à toutes les forces politiques et sociales qui partagent ce souci, et en particulier aux groupes et aux personnes qui se trouvent en difficulté ${ }^{16}$.

Certes, nous sommes ici dans l'ordre de la simulation, et il est difficile de parler d'une existence « réelle» et tangible du Landesverband Chance 2000 par exemple. Le sens de la démarche n'en réside pas moins précisément dans la perfection de ce qui n'est qu'une imitation du fonctionnement politique et du respect de ses principes. Et cette dynamique mimétique se poursuit jusqu'au but ultime, les élections elles-mêmes : le parti obtiendra finalement 3206 Erststimmen et 28566 Zweitstimmen, soit $0,058 \%$ des suffrages selon les résultats officiels ${ }^{17}$.

Le rapport au politique est donc paradoxal, tout comme le paradoxe constitue la raison d'être du projet Chance 2000, ce que suggère la suite du préambule :

Chance 2000 interviendra dans tous les points chauds sociaux et politiques : du centre d'accueil de la gare jusqu'à la décision au Bundestag. Chance 2000 agira avec la conviction que les décisions touchant aux valeurs susceptibles d'orienter la société ne peuvent plus être prises au niveau politique ${ }^{18}$.

15 «Chance 2000 - Vereinssatzung », in Christoph SCHLINGENSIEF, Carl HEGEMANN (Hg.), Chance 2000, p. 19-26.

16 «Die mit der wachsenden Arbeitslosigkeit sich vertiefenden Risse im sozialen Gefüge sowie der mit den rasant wachsenden gesellschaftlichen Ausgrenzungen einhergehende Verlust an menschlicher Würde fordern zu politischen, gesellschaftlichen und alltagspraktischen Neuorientierungen heraus, zu denen die Parteien, die sich bisher an der politischen Gestaltung beteiligt haben, aus eigener Kraft keine Impulse geben können. Besorgt um eine gesellschaftlich und politisch stabile Zukunft unseres Landes im nächsten Jahrtausend, versteht sich Chance 2000 als Partei, die allen politischen und gesellschaftlichen Kräften, die ihre Sorge teilen, Hilfe anbietet, insbesondere auch Gruppen und Einzelnen, die sich in Not befinden. ». Voir note 10

17 Source : http://www.bundeswahlleiter.de/de/bundestagswahlen/fruehere_bundestags wahlen/btw1998.html (consulté le 11 mars 2013).

18 «Chance 2000 wird an allen sozialen und politischen Brennpunkten eingreifen: von der Bahnhofsmission bis zur Entscheidung im Bundestag. Tätigwerden wird Chance 2000 in der Gewissheit, dass über Wertorientierungen nicht politisch entschieden werden kann. ». Voir note 10. C'est moi qui souligne. 
L'investissement politique et social est annoncé alors même qu'est mise en doute l'aptitude du politique à organiser la société en fonctions de valeurs. Dans son programme, le parti se définit par ailleurs comme un Nichtwählerpartei (《 parti des non-votants »), c'est-à-dire comme parti susceptible de réintégrer dans le fonctionnement du suffrage universel comme fondement de la démocratie ceux qui ne votent pas ou plus. Mais dans le même temps, le slogan Wähle Dich selbst semble contredire cette aspiration, à travers la confusion électeurs/candidats évoquée plus haut.

L'entreprise « pseudo-partisane » relève ainsi d'une logique permanente de la contradiction, du contre-pied, déclinée à tous les niveaux. En ce sens, elle rejoint les productions de Schlingensief qui sont plus clairement associées aux arts de la scène. Quoique celles-ci fassent intervenir divers éléments (jeu, extraits filmiques, projections diverses, musique, sons etc.), le terme de Gesamtkunstwerk n'est pas approprié pour les caractériser dans la mesure où ces éléments ne fusionnent pas, mais entrent au contraire en collision $^{19}$. La question demeure néanmoins de savoir quel est le sens à donner à cette logique du paradoxe dans le cas précis de Chance 2000. Nous formulerons l'hypothèse d'interprétation suivante : pour recouvrir une signification, l'appel à réinvestir politiquement l'espace public doit être vidé d'un contenu politique, et la contradiction est le moyen de ce mouvement d'évidement. Chance 2000 serait dès lors un parti «en creux », ce que montre la quasi-absence de revendications concrètes dans le programme et dans les rassemblements. Il en résulte une tension constamment maintenue non plus entre la forme et le fond, mais entre la forme et le «non-fond », tension qui annule le principe d'un consensus à atteindre autour d'intérêts communs, au profit d'un plus petit dénominateur qui ne peut être que l'individu lui-même.

Celui-ci est en effet placé au centre du projet, comme en témoigne la récurrence dans le programme des termes Einzelfallwürdigung (« dignité du cas individuel ») et Einzelfallgerechtigkeit (« justice pour le cas individuel »). S'il paraît rechercher la fédération des intérêts, Schlingensief corrige tout autant cette tendance en misant sur la liberté d'action de chacun des participants par rapport au groupe. C'est le sens qu'il faut donner à la formule reprise dans divers documents : «La plus petite unité du peuple est $1 \mathrm{P}$ - un peuple, c'est-à-dire chacun d'entre-nous $»^{20}$. Le Wähle Dich selbst s'adresse bien à l'individu dans sa biographie propre, avant qu'il ne soit considéré comme élément d'un " collectif des exclus », ce qui, précisément, le

19 Voir sur ce point la retranscription d'une discussion entre Claus Philipp, Christian Reder, Franziska Schößler et Teresa Kovacs sous le titre « Christoph Schlingensief - Ein Gesamtkünstler? », in Pia JANKE, Teresa KOVACS (Hg.), Der Gesamtkünstler Christoph Schlingensief, p. 135-146. F. Schößler, évoquant le principe de contradiction chez Schlingensief, renvoie plus volontiers à la réflexion de Brecht sur l'œuvre d'art totale («Anmerkungen zu Mahagonny», 1930).

20 «Die kleinste Einheit von Volk ist $1 \mathrm{~V}$ - ein Volk, also jeder von uns. », in Christoph SCHLINGENSIEF, Carl HEGEMANN (Hg.), Chance 2000, p. 11. 
renverrait à sa condition sociale : «Le but n'est pas que nous prouvions notre autonomie ; chacun doit apporter la preuve de la sienne, chacun doit s'affirmer lui-même. $»^{21}$. L'enjeu est donc de comprendre comment penser ensemble les intérêts de chacun des individus et la mobilisation d'un groupe. Afin de sortir de ce qui semble être une impasse, il est possible de poser autrement cette question, en se demandant dans quelle mesure la revalorisation de l'individu privé peut être le fondement d'un espace public.

\title{
Subversion et réélaboration de l'espace public
}

Dans un essai intitulé «Espace commun ou espace public? L'antagonisme de la communauté et de la publicité », Etienne Tassin développe le principe d'une opposition entre l'idée de communauté et l'épanouissement d'un espace public. Cette distinction permet de comprendre la conception d'un espace d'expression qui sous-tend implicitement le travail de Schlingensief. Le philosophe conçoit en effet la communauté comme une aspiration collective à la fusion dans un corps unique, une tendance à la convergence dont la communion constituerait le terme idéal :

Cette figure paroxystique de la communauté fait valoir la communion comme aboutissement contradictoire de la communication. Elle indique, a contrario, comment l'institution d'un espace public maintient la communauté à distance d'elle-même : ce qui, certes, rapporte les individus les uns aux autres, mais qui dans le même temps les déporte les uns des autres, ce qui les soumet à un régime d'impropriété mutuelle 22 .

Condition sine qua non de la communication, le maintien d'une distance entre les individus est constitutif de l'espace public, son essence résidant dans la notion d'espace « intervallaire » :

\begin{abstract}
Aussi nous faut-il le comprendre non comme celui de l'apprivoisement ou du déloignement qui tient uni ce que la distance sépare, mais au contraire comme ce qui se déploie entre, comme ce qui, dit Hannah Arendt, inter homines est, ce qui sépare les individus, les tient dans une extériorité des uns aux autres et de chacun à l'ensemble $[\ldots]$. Le problème est moins celui de la distance qui sépare que celui du lien qui unit dans la séparation ${ }^{23}$.
\end{abstract}

Penser l'espace public à partir de ce qui distingue les sujets qui y sont actifs permet donc de lire autrement la prééminence de l'individu dans le

21 «Ziel ist nicht, dass wir unsere Selbstständigkeit beweisen, sondern jeder soll seine eigene beweisen, jeder muss sich selber artikulieren. » Christoph SCHLINGENSIEF, « Einer = 1 Volk », 22 mars 1998, in ibid., p. 28. Le terme « unsere » est souligné par Schlingensief luimême.

22 Etienne TASSIN, « Espace commun ou espace public ? L'antagonisme de la communauté et de la publicité », in Eric DACHEUX (éd.), L'espace public, les essentiels d'Hermès, Paris, CNRS éditions, 2008, p. 115-116.

23 Ibid., p. 124. 
discours à la fois politique et artistique proposé par Chance 2000 . La valorisation de ce qui le distingue de l'Autre, le rappel permanent de son unicité peuvent désormais être lus comme la tentative d'une refondation de l'espace public, refondation dont le corrélat est la subversion de ce qui se donne aujourd'hui pour tel.

Avant d'aborder les phénomènes actuels qui caractérisent l'espace public comme espace de communication entre la sphère privée et l'État, l'interrogation sur le théâtre comme « institution » nous ramène à Habermas. Dans Strukturwandel der Öffentlichkeit, le philosophe mentionne en effet le théâtre parmi les agents qui contribuent à construire l'espace public. Il fait partie des structures sociales de la sphère publique au même titre que les salons, les cafés ou encore l'émergence d'un lectorat. Dans les premières décennies du XVIII ${ }^{\mathrm{e}}$ siècle naît un public qui n'est plus seulement constitué des représentants de la haute aristocratie. De ce point de vue, les pays de langue allemande sont paradigmatiques. En effet, cette formation progressive s'opère certes plus tôt en France et en Angleterre, mais les spectateurs «populaires » n'ont alors qu'une fonction de légitimation du pouvoir de ceux qui sont assis dans les loges, par l'acclamation des productions artistiques dont ces derniers assurent l'existence. Cette transformation progressive serait manifeste dans les ordonnances de la police en France à partir de 1660, dans lesquelles il est question non seulement du public aristocratique, mais aussi d'une frange du public du parterre qui doit être protégée des éventuelles bagarres et de l'agitation, ce qui montre a contrario que ce public bourgeois est en train de se former. En Allemagne, cette évolution s'opère plus tard, au milieu du siècle suivant. On rappellera ainsi la fondation à Hambourg en 1766 du Deutsches Nationaltheater, première scène autre que les théâtres de cour, réclamé avec véhémence par Lessing ${ }^{24}$.

Selon le principe de ce lien intime entre l'institution théâtrale et l'espace public à l'heure de sa naissance, Schlingensief prend acte, dans sa démarche, d'une évolution qui conduit aujourd'hui à une disjonction forte entre les deux. On distinguera de ce point de vue deux stratégies.

Certes, l'artiste entretient un rapport particulier, toujours polémique, avec le théâtre comme forme artistique dont la pratique n'a de sens que si elle en remet systématiquement en cause les frontières et les $\operatorname{codes}^{25}$. Si ce rapport correspond à un positionnement tout aussi spécifique envers l'institution théâtrale, une partie importante de ses réalisations s'inscrivent néanmoins au sein de celle-ci. Pousser le théâtre dans ses retranchements nécessite un contact avec les lieux dans lesquels celui est produit, a fortiori lorsque ceux-ci revêtent une dimension identitaire particulièrement forte. En mettant en scène Parsifal à Bayreuth, le metteur en scène joue implicitement le jeu de la

24 Jürgen HABERMAS, L'espace public. Archéologie de la publicité comme dimension constitutive de la société bourgeoise, Paris, Payot, 1978, p. 49-50.

25 Une vision qui rapproche clairement Schlingensief d'Elfriede Jelinek, partenaire de travail (voir la mise en scène du texte Bambiland au Burgtheater en 2003) et de pensée. 
consécration nationale, il nourrit une culture dominante et non pas une contreculture - pour mieux la miner de l'intérieur ${ }^{26}$.

Avec cette stratégie entriste vient, au fil des productions, s'entrecroiser une seconde qui est celle du déplacement, dont relève Chance 2000. Les différentes actions qui constituent cette "pièce de théâtre illimitée à laquelle chacun peut participer $»^{27}$ se situent pour une part en dehors du cadre théâtral, dans l'espace public au sens physique du terme cette fois : la rue, le métro, le Wolfgangssee ${ }^{28}$.

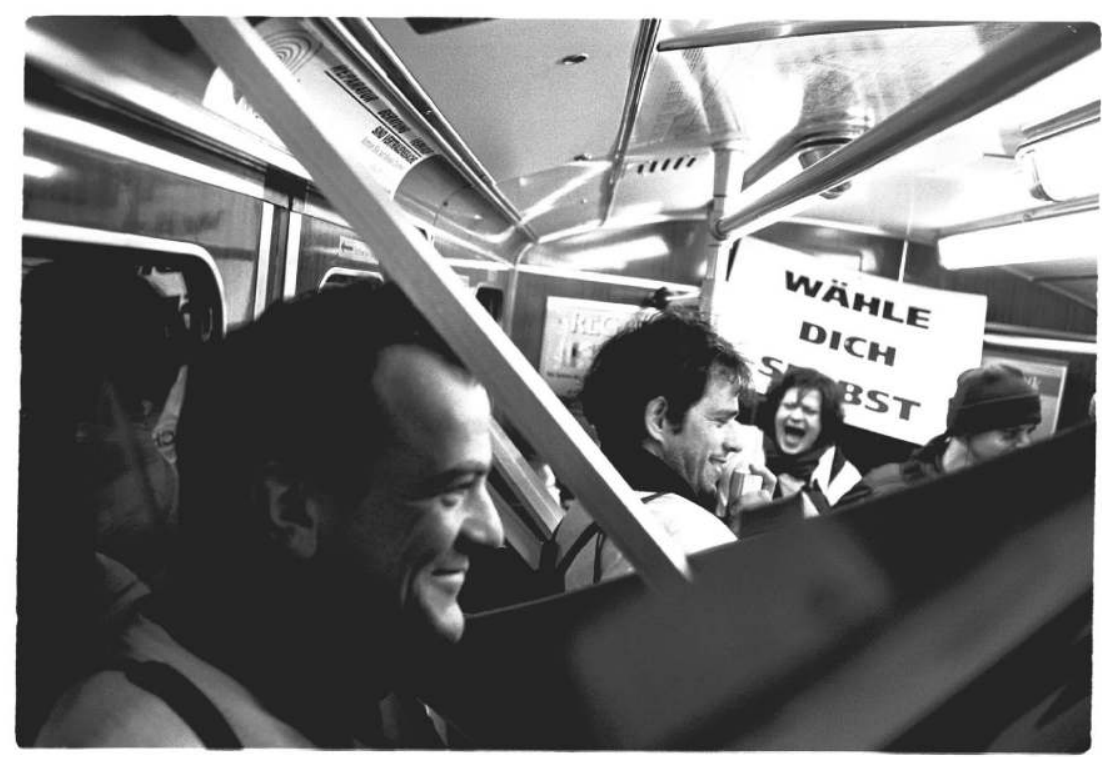

Théâtralisation politisée de l'espace public : le métro berlinois revisité.

Chance 2000. Wahlkampf in Deutschland de Christoph Schlingensief (1998).

(C) Thomas Aurin.

Une telle stratégie va de pair avec la mise en cause du théâtre dans sa pertinence sociale. Dans une de ces démarches d'investissement, Schlingensief engage par exemple les comédiens à se rendre au Kadewe et à s'y comporter de manière anormale : " C'est plus excitant que le Stadttheater, dans lequel c'est au fond Helmut Kohl qui signe toutes les mises en scène $»^{29}$.

26 Rappelons que Schlingensief avait également conçu le pavillon allemand à la biennale de Venise, inauguré le 1er juin 2011.

27 " Chance 2000 erweist sich als ein in jeder Hinsicht unbegrenztes Theaterstück, in dem alle mitmachen können.» Expression empruntée au site consacré à l'artiste : http://www.schlingensief.com/projekt.php?id=t054 (consulté le 11 mars 2013).

28 Outre Chance 2000, il faut bien sûr évoquer de ce point de vue 1'action Bitte liebt Österreich de 2000.

29 « Das ist spannender als Stadttheater, in dem letztlich jede Inszenierung von Kohl stammt. » Christoph SCHLINGENSIEF, Carl HEGEMANN (Hg.), Chance 2000, p. 17. 
Ailleurs, il pose le principe d'une prise de distance comme condition d'une renaissance : « Nous quittons la salle de théâtre et redonnons vie au théâtre ${ }^{30}$. Cette formule dit en condensé le rapport ambigu de l'artiste à l'institution théâtrale : d'un côté, il est nécessaire d'abandonner la scène traditionnelle, de l'autre de contribuer à sa régénération. Le terme employé ici par Schlingensief est celui de « Theaterraum ». Il s'agit donc bien de laisser derrière soi un lieu de culture qui ne serait plus agent de l'espace public dans le sens d'Habermas, pour investir les espaces publics physiques ou du moins ceux qui se donnent aujourd'hui comme tels.

Deux évolutions contemporaines dans la constitution de l'espace public seront ici abordées, dans la mesure où elles occupent une place fondamentale dans le questionnement que propose Schlingensief avec Chance 2000. Il s'agit d'une part de la distinction public/privé, qui permet de penser ensemble l'espace public dans le sens de Habermas et l'espace public physique des urbanistes, et d'autre part du passage d'un espace public à un espace public médiatisé, dans le sens que Dominique Wolton donne à ce terme.

\section{Espace public, espace privé}

L'idée d'une interpénétration des domaines publics et privés au sein de l'espace public n'est pas nouvelle: Habermas la diagnostique à la fin du XIX siècle en constatant une «' fuite' de l'État hors du droit public, c'est-à-dire le transfert de certaines tâches, incombant à l'administration publique, à des entreprises, des établissements, des associations, et des chargés d'affaire relevant du droit privé $"{ }^{31}$. Une telle tendance semble on ne peut plus actuelle, que l'on pense aux agences américaines de sécurité employées pour le maintien de l'ordre en Irak à la place ou à côté de l'armée. Dans un contexte différent, Schlingensief place le problème à un autre niveau qui n'est plus d'ordre juridique, mais symbolique, ce que montre l'action Baden im Wolfgangssee. Dans cet événement, organisé le 2 août 1998, le metteur en scène et son équipe invitent les 4 millions de chômeurs en Allemagne à venir dans la petite ville salzbourgeoise de Sankt Gilgen se baigner dans le lac au bord duquel Helmut Kohl passe ses vacances, l'objectif étant de faire déborder celui-ci et de provoquer l'inondation de la maison du Chancelier. Dans sa forme, ce mélange étrange entre dimension politique, ludique et performative rappelle certains collectifs dont l'importance s'est étendue ces dernières années par le biais d'internet ${ }^{32}$, considérés eux-mêmes comme des p. 55 .

30 «Wir verlassen den Theaterraum und geben dem Theater das Leben zurück. »Ibid.,

31 Jürgen HABERMAS, L'espace public, p. 158.

32 Les collectifs les « dégonflés 》 à Paris, «flagadas » à Bruxelles, «Raplaplas 》 à Lyon, " mous de la roue » à Lille appellent à dégonfler les pneus des $4 \times 4$ afin d'attirer l'attention sur la pollution de l'air. Le «Clan du néon » fédère de manière informelle des groupes apparus en 
animateurs nouveaux de l'espace public. Dans son matériau cette fois, à savoir la personne du Chancelier, Baden im Wolgangssee s'inscrit entre autres dans le sillage de la performance Mein Filz, mein Fett, mein Hase dans le cadre de la documenta $X$ en 1997 : 1'artiste avait alors posé devant l'Orangerie une pancarte sur laquelle était écrit le slogan "Tötet Helmut Kohl », ce qui lui avait valu une arrestation.

Il faut pourtant voir dans ce bain collectif autre chose qu'une simple provocation, dès lors qu'on le met en perspective avec la réflexion sur l'individu et le groupe qui sous-tend l'ensemble du projet. La raison d'être de l'entreprise est en effet à chercher dans le lien qui peut être établi entre personnalisation et déperdition de l'espace public. En effet, la distinction claire entre la sphère privée et l'État constitue le fondement sur lequel s'opère l'émergence de cet espace intermédiaire. Or, le diagnostic qui sous-tend la démarche de Schlingensief est celui non plus simplement d'une personnalisation du pouvoir, mais bien du politique en soi. L'objet du débat public change, il n'est pas l'homme politique mais l'individu dont la sphère privée devient objet spectaculaire. Le geste critique rejoint donc l'absence de revendication mise en évidence plus haut. En venant se baigner chez lui, Schlingensief ne se confronte pas à Kohl sur un projet de société, même s'il rejette clairement les grandes orientations gouvernementales, mais investit la sphère privée, donnée d'ordinaire en spectacle selon le principe « un homme comme vous et moi », dans une sorte de retour à l'envoyeur.

Une autre performance, qui sera ici évoquée plus rapidement, permet d'éclairer et de compléter l'approche artistique de cette problématique public/privé. Il s'agit d'Hotel Prora, action qui débute le 15 mai 1998. Durant neuf jours, trente «clients » par «nuitée » d'un hôtel imaginaire vivent et campent ensemble, dans un confort très relatif. L'expérience est censée permettre à chacun de ces volontaires de mesurer sa sociabilité : les participants doivent se supporter les uns les autres, vivre ensemble. Quelques comédiens professionnels sont présents, et jouent les rôles d'employés de l'hôtel. Durant la journée sont organisées des sorties pour des interventions publiques diverses dans la rue : placardage, cortège, mais aussi simulation d'agitation d'extrême droite à Kreuzberg. Dans le cadre de «l'hôtel », les participants sont par ailleurs observés en permanence par un «public», puisqu'ils sont placés derrière des grilles.

La dissolution problématique entre privé et public est ici déclinée sur plusieurs plans. Le premier se situe dans l'imitation du modèle popularisé par la « télé-réalité ». Un groupe de «citoyens lambda», réunis dans un espace clos, fait l'objet du regard permanent de « spectateurs ». Schlingensief détourne ce schéma en ramenant l'équipement au minimum : les conditions de vie spartiates s'opposent au pseudo-confort standardisé qui caractérise le fameux Loft de l'émission Big Brother ainsi que tous ses dérivés ultérieurs.

2007 dont les actions consistent, de nuit, à éteindre les néons des magasins pour lutter contre le gaspillage de l'électricité. 
Les relations interpersonnelles constituent la seconde déclinaison de cette problématique. L'expérimentation de la vie en commun revient en effet à éprouver les limites des intérêts privés. Enfin, par l'alternance entre les activités politiques et les moments passés dans l'hôtel, les participants conforment une forme physique à une oscillation entre public et privé.

L'intérêt des actions orchestrées par Schlingensief réside dans le fait qu'elles font se rejoindre les deux définitions de l'espace public. Tout comme s'estompe aujourd'hui la distinction claire entre privé et public comme condition de la critique du pouvoir, le devenir de la délimitation entre lieux privés et lieux publics constitue une préoccupation majeure de l'urbanisme contemporain. Ces lieux publics sont la rue, la place, le parvis, le parc. Régénérant ainsi l'espace public «traditionnel », Schlingensief réinvestit ces espaces. Ceux-ci ne sont pas des acteurs institués de la sphère publique au sens où l'entend Habermas. Ils n'en forment pas moins un cadre dans lequel s'épanouit l'espace public comme communication sur l'État. En investissant des lieux publics non théâtraux, Schlingensief réactive donc leur fonction de cadre physique de l'expression et du débat collectifs.

Outre ces lieux publics traditionnels cependant, le philosophe Thierry Paquot rappelle que la conception de "l'espace public » dans la littérature touchant à l'urbanisme contemporain, intègre aussi - non sans critique,

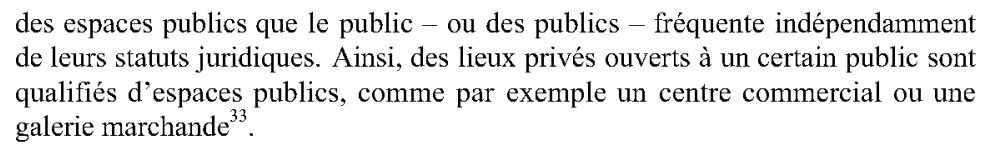

L'expédition organisée au Kadewe répond à cette analyse, dans la mesure où l'ancienne vitrine de l'Ouest fait partie de cette catégorie nouvelle d'espaces «pseudo-publics».

Le 27 mars 1998, Schlingensief se rend au célèbre grand magasin suivi d'une petite troupe arborant le tee-shirt « Chance 2000 ». Le journaliste Robin Detje décrit ainsi l'expédition :

\begin{abstract}
Désormais, le mot d'ordre est : "Pas de violence en faisant les courses! 》. Devant le Kadewe, la troupe de Chance 2000 , forte d'environ trente à quarante personnes, déclenche malgré tout une vive agitation chez le personnel de surveillance du magasin. Tandis qu'Helga s'achète un rouge à lèvres au rez-dechaussée, tout un régiment de brutes embauchées par le magasin s'avance déjà, et explique que l'on dispose ici du droit d'expulser les indésirables, et que l'on fera dès à présent volontiers usage de ce droit sans justification. Impassible, le groupe prend maintenant la direction du premier étage : Achim a besoin d'un pull-over. Christoph Schlingensief compte maintenant venir chaque semaine, un jour fixe que Chance 2000 consacrera à ses achats, et considère que le parti a atteint ses objectifs politiques durant cette sortie shopping: chez Helga, habitante sans emploi d'un foyer, qui n'a jamais possédé de rouge à lèvres, ont été éveillés
\end{abstract}

33 Thierry Paquot, L'espace public, Paris, La Découverte, 2009, p. 3. 
des besoins nouveaux, elle dépensera désormais davantage d'argent. Instaurer l'achat en grand magasin au lieu d'incendier les grands magasins : Chance 2000 apporte ainsi son aide à l'économie allemande ${ }^{34}$.

L'emploi du terme allemand Hausrecht pour désigner le droit d'interdire l'accès au magasin révèle qu'il s'agit bien d'un espace privé. Si Schlingensief fait scandale, c'est davantage dans l'étymologie de ce terme qu'il faut trouver le sens de cette intervention collective. Le terme grec dont est dérivé le scandalum latin («achoppement, ce qui provoque la chute ») désigne le piège posé sur le chemin. Il s'agit de tendre un piège à l'autre, afin de provoquer la chute d'une construction, afin de permettre le dévoilement. Le personnel de sécurité vient mettre un terme involontaire à l'illusion selon laquelle le centre commercial serait aussi un lieu de communication et d'échange, un lieu ouvert, un lieu public. Il révèle la vérité de celui-ci, juridiquement et économiquement privé, dont peut être exclu l'Autre.

\section{Espace public et visibilité}

Dans sa version contemporaine, l'espace public se caractérise par la place qu'y occupent les médias et la façon dont ils en déterminent le fonctionnement, mutation qui conduit Dominique Wolton à substituer à l'expression «espace public » celle «d'espace public médiatisé » ${ }^{35}$. S'il se garde de ne penser ce glissement qu'en termes négatifs, c'est-à-dire en le concevant exclusivement comme un appauvrissement du débat, les formes les plus contemporaines conduisent néanmoins selon lui à un certain nombre de contradictions et de dysfonctionnements. Par ses interventions dans des médias non strictement politiques ${ }^{36}$, Schlingensief prend acte de cette évolution à travers une recherche subversive de visibilité dont il convient de

34 «Die Parole lautet nun: „Keine Gewalt beim Einkaufen!“ Trotzdem führt die Zusammenrottung der vielleicht dreißig- oder vierzigköpfigen „Chance 2000"- Gruppe vor dem Kadewe zu hektischen Aktivitäten der Kaufhaus-Wachleute. Schon während Helga im Erdgeschoß einen Lippenstift kauft, marschiert ein ganzer Trupp von eingestellten Schlägertypen auf und erklärt, dass man hier das Hausrecht habe und von seinem Recht, alle hinauszuwerfen, nun ganz gerne auch ohne Begründung Gebrauch machen würde. Ungerührt zieht die Gruppe weiter in den ersten Stock: Achim braucht einen Pullover. Christoph Schlingensief will jetzt jede Woche hierherkommen, zu seinem Chance-2000-Shopping-Jourfixe, die Partei habe auf ihrem Einkaufsbummel ihre politischen Ziele erreicht: in der arbeitslosen Heimbewohnerin Helga, die noch nie einen Lippenstift besessen habe, seien neue Bedürfnisse geweckt worden, sie werde nun mehr Geld ausgeben. Kaufhauskaufstiftung statt Kaufhausbrandstiftung: So hilft „Chance 2000“ der deutschen Wirtschaft. » Robin DETJE, article paru dans la Berliner Zeitung du 31 mars 1998, cité dans Christoph SCHLINGENSIEF, Carl HEGEMANN (Hg.), Chance 2000, p. 37.

35 Dominique WOLTON, «Les contradictions de l'espace public médiatisé », in Eric DACHEUX (éd.), L'espace public, p. 31-60.

36 Outre son apparition dans des talks shows divers, Schlingensief a lui-même conçu les émissions Talk 2000 sur la chaîne RTL, U 3000 sur MTV (2000), et Freakstars 3000 sur Viva (2002). 
préciser l'enjeu. L'idée de "visibilité », selon Tassin, est en effet consubstantielle à celle d'espace public :

\begin{abstract}
Le domaine public est cet espace de visibilité, lieu de l'apparition du monde : non pas ce sans quoi le monde ne serait pas, mais ce sans quoi il ne pourrait apparaître comme monde commun. Aussi le sens ultime du « vivre ensemble » politique ne peut-il se saisir depuis la question de l'être-en-commun, question de la communauté, mais depuis celle de l'apparaître commun des êtres, question de la polis, du domaine public de visibilité ${ }^{37}$.
\end{abstract}

Dès lors que l'espace n'est public que s'il est celui du dé-loignement, de la distance intersubjective, il s'agit de rendre visible l'altérité de l'un à l'autre. C'est le sens à donner à l'apparition publique sous la forme de la manifestation dans la rue, des actions, mais aussi de la visibilité médiatique. En jouant le jeu des médias, en assumant leur place essentielle dans la publicité (au sens d'Öffentlichkeit), Schlingensief intègre cette dimension, qui sous-tend la dichotomie fondamentale entre ce qu'il appelle le «système 1 » et le «système 2 »: le premier est constitué de toutes les instances et acteurs sociaux qui occupent l'espace de la publicité : institutions, partis politiques, églises, syndicats, monde de l'économie, médias; le second rassemble tout ce qui en est l'opposé selon le critère de l'invisibilité, c'est-à-dire la réalité concrète de la vie sociale. L'enjeu n'est pas tant de nier, sous le double prétexte de leur omniprésence et de leur propension à construire la réalité, la légitimité des agents du système 1 que de réinvestir, pour ceux qui en sont exclus, l'espace public de la visibilité. Pour autant, il s'agit d'éviter d'en devenir l'objet : "Comment redevenir visible sans que le système 1 he les absorbe ? ${ }^{38}$ " En tentant, précisément, d'interrompre sans cesse la production de signification, en maintenant actif le principe de contradiction mis en évidence comme principe fondamental de ce parti qui n'en est pas un ${ }^{39}$.

On peut y voir la forme radicale d'une éducation politique, dans la mesure où elle précède la formulation de contenus. Quel qu'il soit (chômeur, sansabri, handicapé), l'exclu est appelé à prendre conscience de sa propre existence comme être politique et à réinventer l'espace public. Ainsi, le principe de la Selbstdarstellung ( « représentation de soi ») est-il toujours lié à celui de la Selbstverantwortung ( responsabilité pour soi ») dans le programme. Schlingensief le formule ainsi : "Il s'agit d'un parti des individus entièrement autonomes. Je ne suis pas là pour faire de la thérapie.$^{40} \gg \mathrm{Ou}$, sur la forme d'une dernière injonction renvoyant l'individu à

37 Etienne TASSIN, « Espace commun ou espace public », p. 128.

38 «Wie können sie wieder sichtbar werden, ohne daß das System 1 sie schluckt? 》 Mark SIEMONS dans la Frankfurter Allgemeine Zeitung du 16 mars 1998, cité dans Christoph SCHLINGENSIEF, Carl HEGEMANN (Hg.), Chance 2000, p. 48.

39 Ibid., p. 48.

40 «Es ist eine Partei der absolut autonomen Einzelnen. Ich bin nicht da zum Therapieren », in: Christoph SCHLINGENSIEF, «Einer =1 Volk », p. 29. 
son rôle sans pour autant tenter d'en orienter l'expression : «Prenez l'antenne ! Faites quelque chose! N'importe quoi. L'essentiel, c'est que vous puissiez l'assumer ». 